\title{
Impact of internal audit on pacemaker prescription and the immediate costs of pacing in the Northern Region: towards implementation of the recommendations of the British Pacing and Electrophysiology Group
}

\author{
J P Mounsey, S G Ray, M J Griffith, R G Gold, R S Bexton
}

\begin{abstract}
Background-In September 1990 a working party of the British Pacing and Electrophysiology Group recommended the routine use of physiological pacing systems in patients with bradycardia. An audit of the impact of these recommendations on pacemaker prescription in the Freeman Hospital between March 1990 and August 1991 has previously been reported. This paper considers the effect of that internal audit on subsequent pacemaker prescription from September 1991 to February 1993.
\end{abstract}

Patients and methods-The records of 1228 patients who underwent first pacemaker implantation at the Freeman Hospital between March 1990 and February 1993 were audited retrospectively. The patient's age, indication for pacing, pacing mode, and cost of the generator and leads were recorded. The indications for pacing were sinus node dysfunction $(24.9 \%)$ (including patients with coexistent sinus node dysfunction and atrioventricular block), atrioventricular block (57.2\%), atrioventricular block and atrial fibrillation (12.2\%), and carotid sinus syndrome $(5 \cdot 7 \%)$. There was an increase in carotid sinus syndrome $(2 \cdot 7 \%$ to $8 \cdot 1 \%)$ between the two study periods but no other differences in the distribution of case $\mathrm{mix}$ or characteristics of patients. The cost of the working party's recommended optimal pacing mode was calculated from multiplication of the mean cost of the recommended unit over the second half of the study period by the number of patients who would have received that unit.

Results-Between March 1990 and August 1991 atrial pacing for sinus node dysfunction (AAI, AAIR, DDD, or DDDR) increased by $138 \%$ (from $25.0 \%$ to $59.6 \%$ ), mainly because of increased use of AAI mode. Physiological pacing for atrioventricular block (DDD or VVIR) increased by $41 \%$ (from $17 \cdot 0 \%$ to $24 \cdot 0 \%$ ), and VVIR pacing for atrioventricular block with atrial fibrillation increased by $111 \%$ (from $10 \cdot 5 \%$ to $22 \cdot 2 \%$ ). After the internal audit (that is, between September 1991 and February 1993), physiological pacing for atrioventricular block increased by a further $126 \%$ (from $24 \cdot 0 \%$ to $54 \cdot 2 \%$ ). Sixty three per cent of this increase was in the first six months after the internal audit. Pacemaker prescriptions in sinus node dysfunction and atrioventricular block with atrial fibrillation were unchanged (59.6\% physiological pacing for sinus node dysfunction and $22.2 \% v 27 \cdot 3 \%$ VVIR pacing for atrioventricular block with atrial fibrillation). These changes in practice were accompanied by an increase in the age of patients receiving physiological units. Costs of pacemaker hardware for the final six months of the audit (excluding carotid sinus syndrome) increased by $38 \%$ over the costs that would have accrued had pacing policy remained the same as for the initial six month period before the circulation of the recommendations of the working party. Adoption of the guidelines of the working party in full would lead to a further $66 \%$ increase in the costs of hardware.

Conclusions-The principal effect of the recommendations of the working party alone was increased use of $A A I$ pacing for sinus node dysfunction, with little change in the costs of hardware. The internal audit was followed by an increase in physiological pacing for atrioventricular block, and this has had important financial consequences. Internal audit was followed by closer adherence to the recommendations of the working party.

(Br Heart f 1994;71:395-398)

The prescription of pacemakers for bradycardias has been revolutionised by the wide availability of complex units that preserve $\mathrm{AV}$ synchrony and increase the rate of generation of impulses in response to physical activity. Guidelines for pacing of bradycardias that 
take into account these advances in pacemaker technology were published by a working party of the British Pacing and Electrophysiology Group in $1991 .^{1}$ In most cases the guidelines recommended the use of more sophisticated units than previously prescribed and their implementation would lead to an important change in the practice of many British pacemaker centres. ${ }^{23}$ The guidelines would also have important financial implications both in the initial outlay for hardware and in increased costs of follow up.

We have recently reported the results of an audit of the impact of the guidelines of the working party on pacemaker prescription in the Northern Region between March 1990 and August 1991. ${ }^{2}$ This audit documented changes in practice after the initial circulation of the guidelines within the regional cardiothoracic centre in September 1990 and the effect of these changes on expenditure for pacing hardware. The audit was presented internally in September 1991. No specific recommendations for local changes in prescription of pacemakers were made. We report here the effects of this internal audit on subsequent prescription of pacemakers from September 1991 to February 1993. The results of the initial audit are included for comparison.

\section{Patients and methods}

These have been described in detail in our previous report. ${ }^{2}$ Briefly, the records of all patients undergoing a first implantation of a permanent pacing system in the Northern Regional Cardiothoracic Centre at the Freeman Hospital, Newcastle upon Tyne between 1 March 1990 and 28 February 1993 were audited retrospectively. The age, indication for pacing, pacing mode, and the cost of the pulse generator and lead (exclusive of value added tax) were recorded. The costs used were those current in August 1991 for the initial 18 month period and those current in February 1993 for the second 18 month period. The cost of pacing with the optimal mode recommended by the working party were derived as described in our previous report, by multiplying the mean cost of the recommended unit over the second 18 month period by the number of patients who would have received the unit had the recommendations been followed.

Patients were grouped according to the time of pacemaker insertion into six periods of six months, beginning 1 March 1990 and ending 28 February 1993. The guidelines of the working party were published between periods 1 and 2 and the results of the audit of the initial 18 month period were reported internally between periods 3 and 4 .

\section{Results}

Over the three year study period 1228 patients underwent first pacemaker implantation, 550 in the first 18 months, and 678 in the second 18 months. The indications for pacing were sinus node dysfunction (including patients with coexistent sinus node dysfunction and atrioventricular block, $24.9 \%$ ), atrioventricular block $(57 \cdot 2 \%)$, atrioventricular block with atrial fibrillation $(12 \cdot 2 \%)$, and carotid sinus syndrome $(5 \cdot 7 \%)$. The proportion of patients in each diagnostic group remained more or less constant over the three year study period except for an increase in carotid sinus syndrome from $2 \cdot 7 \%$ in the first 18 month audit to $8.1 \%$ in the second 18 month audit. This increase was coincident with the appointment of a physician with an interest in the condition between periods 2 and 3 . There were no other significant differences in the distribution of cases or characteristics of the patients.

Table 1 shows the percentages of patients in each diagnostic group paced with each mode and the mean (SD) initial cost of hardware for each diagnosis for each of the six month periods. The percentage of patients receiving physiological pacemakers for each period is also shown. Patients included in this category received either the optimal or an alternative pacing mode recommended by the working party with the exception of the inclusion of VVIR mode for atrioventricular block and DDD mode for carotid sinus syndrome.

The main change in pacing practice around the time of the recommendations of the working party (between periods 1 and 3) was, as previously documented, ${ }^{2}$ an increase of atrial pacing sinus node dysfunction. This increased by $138 \%$ ( $25.0 \%$ to $59.6 \%)$, mainly because of increased use of AAI mode. There was little change in the use of AAIR mode but there was an increase in dual chamber pacing (DDD and DDDR modes). The recommendations made less impact on practice in atrioventricular block and atrioventricular block with atrial fibrillation. Physiological pacing in atrioventricular block (DDD and VVIR modes) increased by $41 \%(17.0 \%$ to $24.0 \%$ ) and, although the proportional increase in VVIR pacing in atrioventricular block with atrial fibrillation was large at $111 \%$, only $22 \cdot 2 \%$ of patients received the optimal mode in period 3.

After the internal audit was presented (between periods 3 and 6) the most significant change in pacing practice was in atrioventricular block. Physiological pacing increased by $126 \%(24 \cdot 0 \%$ to $54 \cdot 2 \%)$, mainly because of increased use of DDD mode. Half of this increase $(63 \%)$ was in the first six months after the audit (between periods 3 and 4). Atrial pacing in sinus node dysfunction and VVIR pacing in atrioventricular block with atrial fibrillation were unchanged in the second audit.

The changes in pacing practice over the three year study period are reflected by increased use of complex pacing modes in older patients. Table 2 shows the mean (SD) age of patients receiving physiological and non-physiological pacemakers (excluding patients with carotid sinus syndrome) for periods 1,3 , and 6 . In sinus node dysfunction and in atrioventricular block there was an 
Table 1 Percentages of patients in each diagnostic group paced with each mode in each period and mean (SD) cost for each diagnosis in each period

\begin{tabular}{|c|c|c|c|c|c|c|c|c|c|c|}
\hline Period & No & $V V I$ & $V V I R$ & $A A I$ & AAIR & $D D D$ & $D D D R$ & $D D I$ & "Physiological" & Cost \\
\hline \multicolumn{11}{|c|}{ SND/AVB: } \\
\hline 1 & 36 & $69 \cdot 4$ & $5 \cdot 6$ & 13.9 & - & $5 \cdot 6$ & $5 \cdot 6$ & - & $25 \cdot 0$ & $925(438)$ \\
\hline 2 & 56 & $48 \cdot 2$ & - & $28 \cdot 6$ & $5 \cdot 4$ & $14 \cdot 3$ & 3.6 & - & $51 \cdot 8$ & $978(515)$ \\
\hline 3 & 62 & $40 \cdot 3$ & - & 30.6 & $8 \cdot 1$ & 12.9 & 1.6 & 6.5 & 59.6 & $1056(484)$ \\
\hline 4 & 45 & 40.0 & $2 \cdot 2$ & $24 \cdot 4$ & $2 \cdot 2$ & $17 \cdot 8$ & $11 \cdot 1$ & $2 \cdot 2$ & $57 \cdot 8$ & $1111(486)$ \\
\hline 5 & 60 & 40.0 & $3 \cdot 3$ & $28 \cdot 3$ & $3 \cdot 3$ & 16.6 & 6.7 & $1 \cdot 7$ & $56 \cdot 7$ & $1116(496)$ \\
\hline 6 & 47 & 40.0 & $4 \cdot 3$ & $40 \cdot 0$ & $2 \cdot 1$ & $10 \cdot 6$ & & $2 \cdot 1$ & $59 \cdot 6$ & $1092(439)$ \\
\hline \multicolumn{11}{|l|}{ AVB: } \\
\hline 1 & 106 & 83.0 & 3.8 & - & - & $12 \cdot 3$ & 0.9 & - & $17 \cdot 0$ & $821(554)$ \\
\hline 2 & 98 & $81 \cdot 6$ & $3 \cdot 1$ & - & - & $15 \cdot 3$ & - & - & $18 \cdot 4$ & $839(49)$ \\
\hline 3 & 125 & 76.0 & $4 \cdot 8$ & - & - & $19 \cdot 2$ & - & - & $24 \cdot 0$ & $913(509)$ \\
\hline 4 & 115 & $60 \cdot 8$ & 3.5 & - & - & $35 \cdot 6$ & - & - & $39 \cdot 1$ & $1111(590)$ \\
\hline 5 & 114 & $54 \cdot 4$ & 0.9 & - & - & $44 \cdot 7$ & - & - & $45 \cdot 6$ & $1214(636)$ \\
\hline \multirow{2}{*}{\multicolumn{11}{|c|}{ AVB + AF: }} \\
\hline & & & & & & & & & & \\
\hline 1 & 19 & $89 \cdot 5$ & 10.5 & - & - & - & - & - & 10.5 & $726(320)$ \\
\hline 2 & 15 & $93 \cdot 3$ & 6.7 & - & - & - & - & - & $6 \cdot 7$ & $657(226)$ \\
\hline 3 & 18 & $77 \cdot 8$ & $22 \cdot 2$ & - & - & - & - & - & $22 \cdot 2$ & $895(519)$ \\
\hline 4 & 35 & $85 \cdot 7$ & $14 \cdot 3$ & - & - & - & - & - & $14 \cdot 3$ & $813(356)$ \\
\hline 5 & 41 & 82.9 & $17 \cdot 1$ & - & - & - & - & - & $17 \cdot 1$ & $822(352)$ \\
\hline 6 & 22 & $72 \cdot 7$ & $27 \cdot 3$ & - & - & - & - & - & $27 \cdot 3$ & $990(456)$ \\
\hline \multicolumn{11}{|l|}{ CSS: } \\
\hline 1 & 2 & $100 \cdot 0$ & - & - & - & - & - & - & - & $530(28)$ \\
\hline 2 & 2 & - & - & $50 \cdot 0$ & - & $50 \cdot 0$ & - & - & $50 \cdot 0$ & $2260(389)$ \\
\hline 3 & 11 & $9 \cdot 1$ & - & $9 \cdot 1$ & - & $9 \cdot 1$ & - & $72 \cdot 7$ & $81 \cdot 8$ & $1592(441)$ \\
\hline 4 & 9 & $22 \cdot 2$ & - & - & - & - & - & $88 \cdot 8$ & $88 \cdot 8$ & $1587(456)$ \\
\hline 5 & 18 & $5 \cdot 5$ & - & - & - & $4 \cdot 1$ & - & $83 \cdot 4$ & $87 \cdot 5$ & $1794(278)$ \\
\hline 6 & 28 & $3 \cdot 6$ & 3.6 & - & - & - & 一 & 92.8 & $92 \cdot 8$ & $1848(268)$ \\
\hline
\end{tabular}

AVB, atrioventricular block: AVB + AF, atrioventricular block plus atrial fibrillation: CSS, carotid sinus syndrome: Cost, cost of hardware for each diagnosis (exclusive of value added tax) for each period; No, total number of patients in each diagnostic group for each period; physiological, percentage of patients in each diagnostic group in each period receiving physiological pacemakers as defined in the text; SND/AVB, sinus node disease including sinus node disease with atrioventricular block.

increase in the mean age of patients receiving physiological systems. For atrioventricular block the mean age of patients receiving nonphysiological systems also increased. As the mean age of the whole population was stable, this would suggest that only very elderly patients received simple units in period 6 . There was a similar upward trend in the mean age of patients receiving VVIR units for atrioventricular block with atrial fibrillation but this was confused by the higher mean age of the group paced for this diagnosis in period 3.

Implantation of pacemakers for carotid sinus syndrome increased dramatically over the three year study period. Most of these patients received physiological units after period 2. There were no notable changes in practice since the internal audit.

The changes in pacing practice documented in the current audit have been accompanied by a large increase in expenditure on pacemaker hardware. The total cost of hardware for period 6 excluding carotid sinus syndrome was $£ 255872$ ( $£ 46775$ for sinus node dysfunction, $£ 188347$ for atrioventricular block, and $£ 20750$ for atrio-

Table 2 Mean (SD) age of patients receiving physiological and non-physiological pacemakers

\begin{tabular}{|c|c|c|c|c|c|c|}
\hline Period & Physiological & No & Others & No & All & No \\
\hline $\begin{array}{l}\text { SND/AVB: } \\
1 \mathrm{~B} \\
3 \\
6 \\
\text { AVB: }\end{array}$ & $\begin{array}{l}63 \cdot 7(11 \cdot 0) \\
66 \cdot 2(15 \cdot 1) \\
74 \cdot 1(6 \cdot 4)\end{array}$ & $\begin{array}{r}9 \\
37 \\
28\end{array}$ & $\begin{array}{l}72 \cdot 6(12 \cdot 8) \\
72 \cdot 2(16 \cdot 0) \\
74 \cdot 5(16 \cdot 5)\end{array}$ & $\begin{array}{l}27 \\
25 \\
19\end{array}$ & $\begin{array}{l}70 \cdot 4(12 \cdot 8) \\
68 \cdot 6(15 \cdot 6) \\
74 \cdot 3(11 \cdot 8)\end{array}$ & $\begin{array}{l}36 \\
62 \\
47\end{array}$ \\
\hline $\begin{array}{c}1 \\
3 \\
6 \\
6\end{array}$ & $\begin{array}{l}57 \cdot 0(11 \cdot 7) \\
65 \cdot 4(9 \cdot 5) \\
68 \cdot 6(12 \cdot 7)\end{array}$ & $\begin{array}{l}18 \\
30 \\
78\end{array}$ & $\begin{array}{l}77 \cdot 0(11 \cdot 8) \\
77 \cdot 5(11 \cdot 1) \\
81 \cdot 9(7 \cdot 1)\end{array}$ & $\begin{array}{l}88 \\
95 \\
66\end{array}$ & $\begin{array}{l}73 \cdot 6(14 \cdot 4) \\
74 \cdot 6(10 \cdot 9) \\
74 \cdot 7(12 \cdot 0)\end{array}$ & $\begin{array}{l}106 \\
125 \\
144\end{array}$ \\
\hline $\begin{array}{c}\text { AVB + AF: } \\
1 \\
3 \\
6\end{array}$ & $\begin{array}{l}49 \cdot 5(37 \cdot 5) \\
69 \cdot 5(12 \cdot 9) \\
59 \cdot 5(14 \cdot 8)\end{array}$ & $\begin{array}{l}2 \\
4 \\
6\end{array}$ & $\begin{array}{l}73 \cdot 7(9 \cdot 4) \\
83 \cdot 3(5 \cdot 9) \\
76 \cdot 9(9 \cdot 8)\end{array}$ & $\begin{array}{l}17 \\
14 \\
16\end{array}$ & $\begin{array}{l}71 \cdot 1(14 \cdot 7) \\
80 \cdot 2(9 \cdot 6) \\
72 \cdot 1(13 \cdot 6)\end{array}$ & $\begin{array}{l}19 \\
18 \\
22\end{array}$ \\
\hline
\end{tabular}

Abbreviations as for table 1 . ventricular block with atrial fibrillation). If this group had been paced with the same proportions of pacing modes as for period 1 the cost of hardware would have been $£ 185620$ $(£ 42015$ for sinus node dysfunction, $£ 126443$ for atrioventricular block, and $£ 17162$ for atrioventricular block with atrial fibrillation). This represents a $38 \%$ increase in the total cost of hardware over the three year study period and a $49 \%$ increase in the cost of pacing atrioventricular block. If the patients in period 6 had been paced with the optimal mode recommended by the working party the costs of hardware would have been $£ 387436$ ( $£ 77438$ for sinus node dysfunction, £273 456 for atrioventricular block, and $£ 36542$ for atrioventricular block with atrial fibrillation). This would represent a $66 \%$ increase in initial expenditure on hardware over and above our current practice.

\section{Discussion}

The Department of Health White Paper, Working for Patients, defined medical audit as the systematic, critical analysis of the quality of medical care, the use of resources, and the resulting outcome and quality of life for the patient. ${ }^{4}$ In practice this may be achieved by the audit cycle, which consists of four steps. ${ }^{5}$ (a) definition of criteria and standards; $(b)$ data collection on current performance; $(c)$ assessment of performance against the criteria and standards; $(d)$ identification of the need to change.

In this report we have shown how completion of an audit cycle has influenced our practice in the prescription of pacemakers for bradycardias. Guidelines for the prescription of pacemakers for bradycardias were established by the working party of the British Pacing and Electrophysiology Group in 
$1991 .{ }^{1}$ Our initial assessment of pacemaker prescription in the Northern Region, ${ }^{2}$ which covered six months before and 12 months after circulation of the guidelines showed that their effect was most notable where it had least financial impact-in increased use of atrial pacing for sinus node dysfunction. The guidelines had much less effect on pacemaker prescription for atrioventricular block and atrioventricular block with atrial fibrillation. The presentation of this audit internally stimulated considerable discussion and the final step in the audit cycle, the need for change, was recognised although not formalised into wholesale adoption of the guidelines of the working party. Assessment of our practice 18 months later indicated a large increase in the prescription of physiological pacemakers for atrioventricular block, which mainly occurred in the six months after presentation of the initial audit. The increase in atrial pacing for sinus node dysfunction was maintained during the second 18 month period but practice for atrioventricular block with atrial fibrillation was essentially unchanged.

The changes in pacemaker prescription documented in the current report have resulted in increased initial cost of pacemaker hardware. There will also be an increase in the cost of long-term follow up because the necessary equipment and technical expertise for the management of complex pacing units is concentrated on one site, at the Freeman hospital. This will necessitate a round trip of over 200 miles for some patients. Older patients are now receiving physiological units and these patients will often require ambulance transport, further increasing the cost.

The Department of Health White Paper emphasised the balance between consumption of resources and improvement in the quality of life. Although adoption of the guidelines of the working party in full would probably enhance the quality of life for most patients, the increase in both short-term and long-term costs would be considerable. There is probably still scope for the more widespread use of physiological pacemakers in our population. We suggest that there is still a case for the use of simple units in a few very immobile, elderly patients who would derive little benefit from rate responsive or dual chamber systems.

In conclusion, the principal effect of the recommendations of the working party of the British Pacing and Electrophysiology Group on pacemaker prescription in the Northern Region was the increased use of atrial pacing for sinus node dysfunction with little change in the cost of hardware. An internal audit of pacemaker prescription 12 months after circulation of the guidelines of the working party was followed by a large increase in physiological pacing for atrioventricular block and this has had major financial implications. Internal audit may therefore influence pacemaker prescription but the cost of this will need to be considered when planning pacing budgets.

1 Report of a working party of the British Pacing and Electrophysiology Group. Recommendations for pacemaker prescription for symptomatic bradycardias. $B$ Heart f 1991;66:185-91.

2 SG Ray, MJ Griffith, S Jamieson, RS Bexton, RG Gold. Impact of the recommendations of the British Pacing and Electrophysiology Group on pacemaker prescription and on the immediate costs of pacing in the Northern region. Br Heart $\mathcal{F}$ 1992;68:531-4.

3 de Belder MA, Linker NJ, Jones S, Camm AJ, Ward DE. Cost implications of the British Pacing and Electrophysiology Group's recommendations for pacing. $B M \mathcal{F}$ 1992;305:861-5.

4 Secretaries of State for Health, England, Wales, Northern Ireland, and Scotland. Working for Patients (Cmd555) London: HMSO, 1989.

5 Irvine DH, Irvine S. Making sense of audit. Oxford: Radcliffe Medical Press. 1991:17-24. 